\section{Human in vitro} Parkin ubiquitin E3 ligase assay

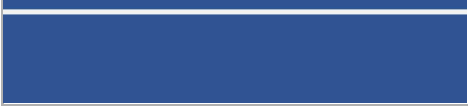

JUL 31, 2021

\section{open ठaccess}

\section{DOI:}

dx.doi.org/10.17504/protocol s.io. bsfrnbm 6

\section{Protocol Citation: Michael} Stevens, Miratul M. K. Muqit 2021. Human in vitro Parkin ubiquitin E3 ligase assay. protocols. io

https://dx.doi.org/10.17504/p rotocols.io. bsfrnbm 6

\section{License: This is an open} access protocol distributed under the terms of the Creative Commons Attribution License, which permits unrestricted use, distribution, and reproduction in any medium, provided the original author and source are credited

Protocol status: Working We use this protocol and it's working

Created: Feb 16, 2021

Last Modified: Dec 26, 2022

PROTOCOL integer ID:

47313

Keywords: ASAPCRN

\section{(3) Human in vitro Parkin ubiquitin E3 ligase assay}

Michael Stevens ${ }^{1}$, Miratul M. K. Muqit ${ }^{1}$

${ }^{1}$ Medical Research Council Protein Phosphorylation and Ubiquitylation Unit, School of Life Science, University of Dundee, Dow Streets, Dundee, DD1 $5 \mathrm{EH}, \mathrm{UK}$

\section{ASAP2020 alessi}

m. muqit 


\section{ABSTRACT}

Mutations in PARK2 encoding Parkin are causal for early-onset Parkinson's disease. Parkin is a ubiquitin E3 ligase and is activated by the PINK1 kinase to stimulate ubiquitin-dependent removal of damaged mitochondria via autophagy (mitophagy). Previous studies have identified Miro1 as a Parkin substrate in cells and in vitro. This protocol describes a facile direct assay of Parkin E3 ligase activity using recombinant untagged Parkin and GST-Miro1 as a substrate. This assay can be employed to assess the effect of small molecule on Parkin activity as well as the impact of Parkinson's disease mutations of Parkin.

In cells the removal of malfunctioning organelles is important for cellular survival. Damaged mitochondria are removed via mitophagy upon activation of the PTEN induced kinase 1 (PINK1) / Parkin pathway. In healthy mitochondria PINK1 is cleaved by PARL and released into the cytoplasm where it is degraded by the N-end rule. In damaged mitochondria depolarisation of the mitochondrial membrane stabilises PINK1, causing it to accumulate on the outer mitochondrial membrane. Stabilised PINK1 phosphorylates Ser65 on both ubiquitin and the ubiquitin like (UbI) domain of Parkin, this activated Parkin. Active Parkin ubiquitinates outer mitochondrial membrane proteins, leading to ubiquitin chains containing pSer65 ubiquitin. The formation of these chains on the mitochondria identifies it for removal by mitophagy. Loss of activity in either Parkin or PINK1 have been observed in cases of early-onset Parkinson's disease. Miro1 is a mitochondrial GTPase involved in mitochondrial morphology and translocation. Previous studies have shown Miro1 to be efficiently ubiquitylated by Parkin at multiple sites.

The following protocol outlines a substrate-based catalytic ubiquitin ligase assay for human Parkin that employs GST-Miro1. Parkin is pre-incubated with either wild type (wt) PINK1 from Tribolium castaneum (TcPINK1) to activate it; the kinase inactive (KI, D359A) version to maintain Parkin's inactive state; or is left untreated. In each case the proteins, UbE1, UbE2L3, Flag-Ub and GST-Miro1 are then added to start the ubiquitylation reaction. The reaction is terminated by the addition of SDS-loading dye. Once the assay is complete, western blotting is performed to demonstrate Parkin activation via multi-monoubiquitylation of GST-Miro1 as seen by 3 or 4 additional bands in the sample treated with wt TcPINK1, but not in the samples treated with KI TcPINK1 or the untreated sample.

\section{ATTACHMENTS \\ Human in vitro Parkin \\ ubiquitin E3 ligase \\ assay.pdf \\ MATERIALS}

\section{Consumables:}


- 8 Hydrochloric acid VWR International Catalog \#20252-335

- 8 Sodium hydroxide Contributed by users Catalog \#795429

- Prepare a 5M solution in deionised water.

- 88 TRIS base VWR International Catalog \#103157P

- Prepare a $1 \mathrm{M}$ Tris- $\mathrm{HCl} \mathrm{pH} 7.5$ stock in deionised water with the $\mathrm{pH}$ adjusted using $37.5 \% \mathrm{HCl}$.

- 8 Sodium chloride VWR International Catalog \#27788.366

- Prepare a $5 \mathrm{M} \mathrm{NaCl}$ stock using deionised water.

-

8 Ethylenediaminetetraacetic acid disodium salt dihydrate (EDTA) Contributed by users Catalog \#E6635-500G

- Prepare a $0.1 \mathrm{M}$ stock at $\mathrm{pH} 7.5$ in deionised water by the addition of $5 \mathrm{M} \mathrm{NaOH}$

- 8 Magnesium chloride hexahydrate Contributed by users Catalog \#M2670 5006

- \& Adenosine Tri-Phosphat (ATP) Abcam Catalog \#ab14730

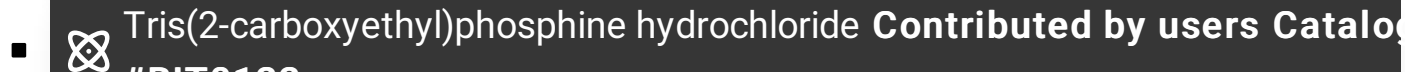
\#BIT0122

- Prepare a $0.5 \mathrm{M}$ stock solution in deionised water.

- $\mathrm{P} 10, \mathrm{P} 200$ and P1000 pipette tips

- 8 Corning microcentrifuge tubes (polypropylene) Contributed by users Catalog \#CLS3620-500EA

- 8 NuPAGE LDS Sample Buffer (4x) Thermo Fisher Scientific Catalog \#NP0007

- His-Ube1

- UbE2L3

- Flag Ubiquitin

- Parkin

- MBP - kinase inactive [D359A] Tribolium castaneum PINK1 [MBP-KI TcPINK1]

- MBP - wild type Tribolium castaneum PINK1 [MBP-wt TcPINK1]

- GST-Miro1 [1-592] - expressed and purified by the method outlined at the end of the protocol. 


\section{Equipment:}

- $20 \backslash u l, 200 \backslash u l$ and $1000 \backslash u$ l Greiner pipettes

- Benchtop cooled microcentrifuge - Thermo Fisher Sorvall Legend Mirco 21r Microcentrifuge

- Eppendorf ThermoMixer - 5382000031 Eppendorf

\section{Buffered Solutions:}

- Kinase Master Mix: 60 mM Tris-HCl pH 7.5, 12 mM MgCl $2,0.12$ mM ATP - Protein concentrations: 0.9 \uM Parkin

- Ubiquitin Master Mix: 50 mM Tris- $\mathrm{HCl} \mathrm{pH} \mathrm{7.5,} 10 \mathrm{mM} \mathrm{MgCl}_{2}, 4 \mathrm{mM}$ ATP and $1 \mathrm{mM}$ TCEP

- Protein concentrations: 2 \UM UbE2L3, 0.24 \uM His-UbE1, 100 \uM Flag-Ub and 1 \UM GST-Miro1

\section{Final concentrations:}

- Kinase reaction: $50 \mathrm{mM}$ Tris- $\mathrm{Hcl}$ pH 7.5, $10 \mathrm{mM} \mathrm{MgCl}_{2}, 0.1 \mathrm{mM}$ ATP, $0.75 \backslash \mathrm{uM}$ Parkin and 0.18 \uM MBP-wt PINK1 \MBP-KI PINK1

- Ubiquitylation reaction: $50 \mathrm{mM}$ Tris- $\mathrm{HCl}$ pH 7.5, $10 \mathrm{mM} \mathrm{MgCl}$, $2.05 \mathrm{mM}$ ATP, 0.375 \uM Parkin, 1 \uM UbE2L3, 0.12 \uM His-UbE1, 50 \uM Flag-Ub, 0.09 \uM MBP-wt PINK1/MBP-KI PINK1 and 0.5 \UM GST-Miro1

- Final reactions protein concentrations: $0.28125 \backslash u M$ Parkin, $0.75 \backslash u M$ UbE2L3, 0.09 \uM His-UbE1, 37.5 \uM Flag-Ub, 0.0675 \uM MBP-wt PINK1/MBP-KI PINK1 and 0.375 \uM GST-Miro1

\section{SAFETY WARNINGS}

(1) For hazard information and safety warnings, please refer to the SDS (Safety Data Sheet).

\section{Preamble}

The set of reactions performed are where Parkin is phosphorylated by wt MBPTcPINK1, kinase 
1 inactive (KI) MBP-TcPINK1, and no MBP-TcPINK1, with all reactions performed in duplicate.

2 \ $12.5 \mu \mathrm{L}$ kinase master mix and \ $15 \mu \mathrm{L}$ ubiquitylation master mix are required per reaction.

3 The volume of master mix made in each case is the volume required for the number of reactions $+10 \%$ rounding up, for instance if 6 reactions are going to be performed then enough master mix should be made for $6+1$ ( 0.6 rounding up) reactions.

\section{Making Master Mixes}

4 Prior to starting reactions keep reagents and master mixes $8^{\circ}$ On ice

5 Combine non-protein reagents for each master mix, vortex the master mix, and settle the master mix to the bottom of the microcentrifuge tube.

6 Defrost proteins $\AA^{\circ}$ On ice and add to kinase and ubiquitin master mixes, mix master mixes by pipetting.

7 Just before starting kinase reactions dilute wt and KI MBP-PINK1 to $2.16 \mathrm{uM}$ with deionised water, aliquot $\triangle 2.5 \mu \mathrm{L}$ of the mixes to two different microcentrifuge tube per MBP-PINK1 mix along with deionised water.

\section{Ubiquitylation Reactions}


8 Begin the kinase reaction by adding $312 \mu \mathrm{L}$ kinase master mix to each reaction tube, and incubate the reaction $\because 1000 \mathrm{rpm}, 37^{\circ} \mathrm{C}, 00: 30: 00$

9 Add \& $15 \mu \mathrm{L}$ ubiquitylation master mix to each reaction and incubate the ubiquitylation reaction

$\because 1000 \mathrm{rpm}, 37^{\circ} \mathrm{C}, 00: 30: 00$. Stop the reaction by the addition of

I $10 \mu \mathrm{L} 4 \mathrm{x}$ LDS loading buffer and incubate at $8^{\circ} 95^{\circ} \mathrm{C}$ for $\circlearrowleft 0: 05: 00$ to denature the proteins and make SDS-PAGE samples.

\section{Resolving Target Proteins by Western Blot}

10 Analyse assays by immunoblotting for the protein read outs GST-Miro1 to observe target ubiquitylation, His-Ube1, and Flag-Ub.

11 Dilute the final reaction sample 1 in 3 with $1 x$ LDS loading buffer and resolve \& $5 \mu \mathrm{L}$ final sample by SDS-PAGE using two $4-20 \%$ polyacrylamide gel.

12 Transfer proteins to nitrocellulose membranes and immunoblot against anti-Miro1, anti-Flag, and anti-6His.

13 If ubiquitylation has worked there should be ubiquitin chains observed with the flag antibody only in reactions with MBP-wt TcPINK1 added.

14 In addition, Miro 1 ubiquitylation should only be observed in reactions with wt TcPINK1 added. 